\title{
LASER ACTION IN STELLAR ENVELOPES
}

\author{
Y.P. VARSHNI and C. S. LAM \\ Department of Physics, University of Ottawa, Canada
}

(Received 19 January, 1976)

\begin{abstract}
It is shown that in high-temperature stars in which high speed mass loss is occurring, the rapidly recombining plasma in the stellar envelope can act as an amplifying medium. Model calculations for laser action in $\mathrm{He}$ II $\lambda .4686$, using the collisional-radiative model, are presented. Menzel's hypothesis of laser action in distended stellar atmospheres is shown to be fully substantiated. The relevance of these results in resolving the problem of intensity anomalies in the spectra of WolfRayet stars is pointed out.
\end{abstract}

\section{Introduction}

We are indebted to Menzel (1970) for a clear discussion of the possibility of a laser action in non-LTE atmospheres. In problems related to gaseous nebulae, Menzel (1937) had introduced a dimensionless parameter $b$ to indicate the degree of departure of a gas from thermodynamic equilibrium at temperature $T_{e}$ of the electron gas. This parameter is unity for thermodynamic equilibrium. Thus, one writes

$$
\frac{N_{k}}{N_{i}}=\frac{b_{k}}{b_{i}} \frac{g_{k}}{g_{i}} \exp \left(-h v_{k i} / k T_{e}\right),
$$

where the quantities $N_{i}$ and $N_{k}$ are the atomic populations of the lower and upper levels, whose respective statistical weights are $g_{i}$ and $g_{k}$.

In arguing for laser action in stars with highly distended atmospheres, Menzel (1970) took the analogy of the behaviour of $b_{k} / b_{i}$ for gaseous nebulae. It was a remarkable extension based on an intuitive insight into the problem. Menzel showed that for laser action to take place,

$$
\left(b_{k} / b_{i}\right) \exp \left(-h v_{k i} / k T_{e}\right)>1 .
$$

In laser physics, one defines a quantity $P$, which is a measure of the population inversion and is given by (Lengyel, 1966)

$$
P=N_{k} / g_{k}-N_{i} / g_{i} \text {. }
$$

We shall find it more convenient to discuss laser action in terms of $P$, rather than in terms of $b_{k} / b_{i}$. For laser action to be operative, $P>0$. This condition is equivalent to that given in inequality (2). The interesting question then arises as to how $P>0$ in the emission line region of stars, where the dilution of stellar radiation is not very appreciable.

In this paper we show that for such high-temperature stars in which high speed mass 
loss is occurring, there can be a population inversion in some atomic species. We shall lay emphasis on the physics of the processes taking place. Model calculations for laser action in the $n=4 \rightarrow n=3$ transition (24686) in He II are presented. Menzel's hypothesis of laser action in stellar atmospheres is shown to be fully substantiated. The relevance of these results in resolving the problem of intensity anomalies in the spectra of Wolf-Rayet stars is pointed out.

A comment on the choice of He II $\lambda 4686$ is in order. The reasons are twofold. Firstly, this line occurs in the spectra of many astronomical objects, and secondly, $\mathrm{He}^{+}$is a hydrogen-like system for which it is feasible to carry out calculations because the necessary cross sections for the various elementary processes (recombination, ionization, excitation, radiative transitions) are available from theory and/or experiment.

\section{The Expanding Envelope}

We consider what happens when the plasma in the outer layers of a star rapidly expands. The essential physics of the problem, though not in the stellar context, was discussed by Gudzenko and Shelepin (1963) and Gudzenko et al. (1966). These authors proposed that if there is sufficiently rapid cooling of the free electrons, there can be a population inversion in the lower levels of an atom, and this can lead to laser action. The subject has been recently reviewed by Gudzenko et al. (1974).

The basic theory for obtaining the properties of a decaying plasma was given by Bates et al. (1962a, b) and is called the collisional radiative (CR) model. A number of workers (Bates and Kingston, 1963; McWhirter and Hearn, 1963; Drawin, 1969; Zemtsov, 1969; Bohn, 1971; Fujimoto et al., 1972), using this model, have carried out calculations on the properties of a rapidly decaying monoatomic plasma. We shall also use the CR model here.

The results obtained from a number of subsequent experiments (Stevefelt and Robben, 1972, and papers quoted therein) for the recombination rates are for the most part in reasonable agreement with those calculated from the $\mathrm{CR}$ model. Experimental confirmation of the prediction regarding the inversion in the atomic level population has been obtained by Hoffmann and Bohn (1972) for hydrogen, and by Irons and Peacock (1974) for $\mathrm{C}^{5+}$.

Results obtained by previous workers and from our own preliminary calculations (Varshni and Lam, 1974) showed that to obtain laser action in He II 24686 , the initial temperature of the plasma has to be of the order of $50000 \mathrm{~K}$, temperatures which are encountered in the Wolf-Rayet stars. Here we shall use the term 'Wolf-Rayet' stars in the general sense, to include both the 'classical' WR stars and the central stars of some planetary nebulae. There now exists ample theoretical and observational evidence that atmospheres of these stars depart seriously from a condition of local thermodynamic equilibrium (LTE). We consider a mass of plasma at the base of the extended atmosphere of a Wolf-Rayet star, roughly where its 'phot osphere' would lie. (In the ordinary sense of the word, of course, one is not even sure whether these stars 
possess a photosphere.) It will be reasonable to assume that the condition there will also correspond to non-LTE. Hence to obtain the relative concentrations of $\mathrm{He}, \mathrm{He}^{+}$ and $\mathrm{He}^{++}$at a particular electron density $\left(n_{e}\right)$ and electron temperature $\left(T_{e}\right)$ we use the non-LTE method of House (1964). There is also a considerable body of observational data which lead to the conclusion that Wolf-Rayet stars and some related early type stars are undergoing a continuous outflow of matter (Ambartsumyan, 1958; Sobolev, 1960; Hack, 1968).

Next, we assume that this plasma expands adiabatically, for which the plasma density $N$ and $T_{e}$ are related by $T_{e} N^{1-\gamma}=$ const. We assume $\gamma=5 / 3$; for the actual plasma the value will be slightly smaller. The flow is supersonic and to a first approximation, the plasma is assumed to be 'frozen' during the rapid fall of temperature, for which a factor of 5 is assumed. (The same factor for the fall in the temperature has been used by Gudzenko et al., 1966.)

This cooled plasma will undergo rapid recombination, and the populations of atomic levels can be obtained from the CR model. For an optically thin, hydrogen-like plasma, the rate at which the population density $n(p)$ of level $p$ changes is given by the differential equation

$$
\begin{aligned}
\dot{n}(p) \equiv \frac{\mathrm{d} n(p)}{\mathrm{d} t}= & \sum_{q=1}^{p-1} C(q, p) n(q) n_{e}- \\
& -\left\{\left[\sum_{q=1}^{p-1} F(p, q)+\sum_{q=p+1}^{\infty} C(p, q)+S(p)\right] n_{e}+\right. \\
& \left.+\sum_{q=1}^{p-1} A(p, q)\right\} n(p)+ \\
& +\sum_{q=p+1}^{\infty}\left[F(q, p) n_{e}+A(q, p)\right] n(q)+ \\
& +\left[\alpha(p) n_{e}+\beta(p)\right] n_{i} n_{e},
\end{aligned}
$$

where $p$ and $q$ are the principal quantum numbers. The meaning of other symbols in Equation (4) are as follows:

$$
n_{l}=\text { ion density, }
$$

$C(q, p)=$ rate coefficient for excitation from level $q$ to $p$ by electronic collision,

$F(p, q)=$ that for de-excitation from level $p$ to $q$,

$S(p)=$ that for ionization from level $p$,

$A(p, q)=$ Einstein coefficient for radiative transition from level $p$ to $q$,

$\alpha(p)=$ rate coefficient for three-body recombination to level $p$,

$\beta(p)=$ that for radiative recombination.

A set of infinite number of the coupled Equations (4) with $p=1,2, \ldots, \infty$ describes the population densities of all the discrete levels. To solve it practically, the following 
two assumptions are used: (1) For all levels $p$ above some high-lying level $r$, the population density $n(p)$ obeys the Saha-Boltzmann distribution law, i.e., these levels are in LTE:

with

$$
n(p)=n_{E}(p) \equiv Z(p) n_{i} n_{e} \text { for } p>r
$$

$$
Z(p)=\left[g(p) / 2 \omega_{i}\right]\left(h^{2} / 2 \pi m k T_{e}\right)^{3 / 2} \exp \left[\chi(p) / k T_{e}\right],
$$

where $g(p)$ and $\omega_{i}$ are the statistical weight of level $p$ and the partition function of ion, respectively, $\chi(p)$ is the ionization potential of level $p$, and $m$ is the electron mass. The population density $n_{E}(p)$ represents that of level $p$ in LTE. We have used $r=10$, while some previous workers have used $r$ as high as 35. As our project involved a large number of calculations, to minimize computer time, we compared the results obtained for population densities for a few sample cases for $r=10$ and $r=25$. It was found that in general the differences are quite small and to the degree of accuracy that we are interested in, can be neglected. (2) The rate equation can be set equal to zero for all levels other than the ground level $(p=1)$

$$
\begin{aligned}
& \mathrm{d} n(1) / \mathrm{d} t \neq 0 \\
& \mathrm{~d} n(p) / \mathrm{d} t=0 \quad \text { for } \quad p=2,3, \ldots, r .
\end{aligned}
$$

Equation (4) can be rewritten in terms of the normalized population density which is defined by $\varrho(p)=n(p) / n_{E}(p)$.

$$
\begin{aligned}
\frac{\dot{n}(p)}{n_{E}(p)}= & \sum_{q=1}^{p-1} F(p, q) \varrho(q) n_{e}-\left\{\left[\sum_{q=1}^{p-1} F(p, q)+\sum_{q=p+1}^{\infty} C(p, q)+\right.\right. \\
& \left.+S(p)] n_{e}+\sum_{q=1}^{p-1} A(p, q)\right\} \varrho(p)+ \\
& +\sum_{q=p+1}^{\infty}\left[C(p, q) n_{e}+\frac{Z(q)}{Z(p)} A(q, p)\right] \varrho(q)+ \\
& +\frac{1}{Z(p)}\left[\alpha(p) n_{e}+\beta(p)\right]=0,
\end{aligned}
$$

with $p=2,3, \ldots, r$. The summations to infinity appearing in equation (8) were cut off at $q=20$.

Assuming the solution of the form

$$
\varrho(p)=r_{0}(p)+r_{1}(p) \varrho(1) \text { with } 2 \leqslant p \leqslant r,
$$

and substituting it into Equation (8), we have two sets of $r-1$ equations for $r_{0}(p)$ and $r_{1}(p)$ with $2 \leqslant p \leqslant r$, because Equation (8) must hold for any value of $\varrho(1)$. The solutions $r_{0}(p)$ and $r_{1}(p)$ obtained from these two sets of equations give the population density

$$
n(p)=Z(p) r_{0}(p) n_{i} n_{e}+[Z(p) / Z(1)] r_{1}(p) n(1)
$$

The coefficients $r_{0}(p)$ and $r_{1}(p)$ are called the population coefficients. 
Extensive calculations of population densities for $n=3$ and 4 levels of He II were carried out for a grid of $n_{e}$ and $T_{e}$ values. The sources for data or expressions for the various rate coefficients entering Equation (4) are as follows. $C(q, p)$ : Drawin (1967); $F(p, q)$ : It can be expressed in terms of $C(q, p)$ by the method of detailed balancing; $S(p)$ : Drawin (1969); $A(p, q)$ : Green et al. (1957); $\alpha(p)$ : Drawin (1967); $\beta(p)$ : Seaton (1959). The initial density of helium atoms before expansion was taken to be $1 \times 10^{14}$ $\mathrm{cm}^{-3}$.

\section{Results and Discussion}

We express our results in terms of $P$, where $P$ is defined by Equation (3). $P$ is related to the fractional gain per unit distance, $\alpha$, at the centre of a Doppler-broadened line by the following expression (cf. Willett, 1974)

$$
\alpha=\left(\frac{\ln 2}{\pi}\right)^{1 / 2}\left[\frac{g_{k} A_{k i}}{4 \pi}\right] \frac{P \lambda_{0}^{2}}{\Delta v},
$$

where $\lambda_{0}$ is the centre wavelength of the transition, and $\Delta v$ is the linewidth. $\alpha$ describes the intensity of a plane wave at $\lambda_{0}$ according to

$$
I=I_{0} e^{\alpha l},
$$

where $l$ is the length over which gain occurs. The results are shown in Figure 1 which shows contours of equal $P$ on a $n_{e}, T_{e}$ plot. The numerical values of $n_{e}$ and $T_{e}$ given on the diagram refer to the plasma after expansion.

It will be noticed from Figure 1 that strong population inversion occurs only within a narrow region of the diagram. On a three-dimensional plot, with $P$ as the third axis, the plot will appear as a mountain with a steep slope on the high $n_{e}$ side. The peak value of $P$ is $\simeq 2.9 \times 10^{6} \mathrm{~cm}^{-3}$ and it occurs at $T_{e} \simeq 20500 \mathrm{~K}$ and $n_{e} \simeq 2.5 \times 10^{15}$ $\mathrm{cm}^{-3}$.

Let us consider some of the numerical magnitudes involved. The electron density in surface layers of the early type stars is estimated to be $\sim 10^{15} \mathrm{~cm}^{-3}$. After expansion, this will be reduced to $\sim 10^{14} \mathrm{~cm}^{-3}$ (Underhill, 1968, also quotes this value as a possible electron density in a typical Wolf-Rayet atmosphere). At this electron density and $T_{e}$ between $15000 \mathrm{~K}$ and $20000 \mathrm{~K}, P \simeq 3 \times 10^{4} \mathrm{~cm}^{-3}$. Assuming $\Delta \lambda=50 \AA$, we get $\alpha \simeq 1.5 \times 10^{-9} \mathrm{~cm}^{-1}$. If we make the reasonable assumption that $l \sim R_{\odot}\left(=7 \times 10^{10} \mathrm{~cm}\right)$, we get $\alpha l \simeq 105$. This value, however, appears to be on the high side, because of the fcllowing two factors. First, we have not taken into account the radiative excitation due to the star and, second, we have assumed that the plasma is optically thin, which is not expected to be the case. Also, the spatial extent of the inversion region is only an educated guess. The present calculations do, however, clearly show that laser action is entirely feasible in the atmospheres of stars which are undergoing mass loss.

What will be the characteristics of emission lines due to laser action produced by the mechanism discussed here? Rapid cooling is possible when there is high speed expansion, thus the lines are expected to be broad. Ordinarily the intensity of a line will 


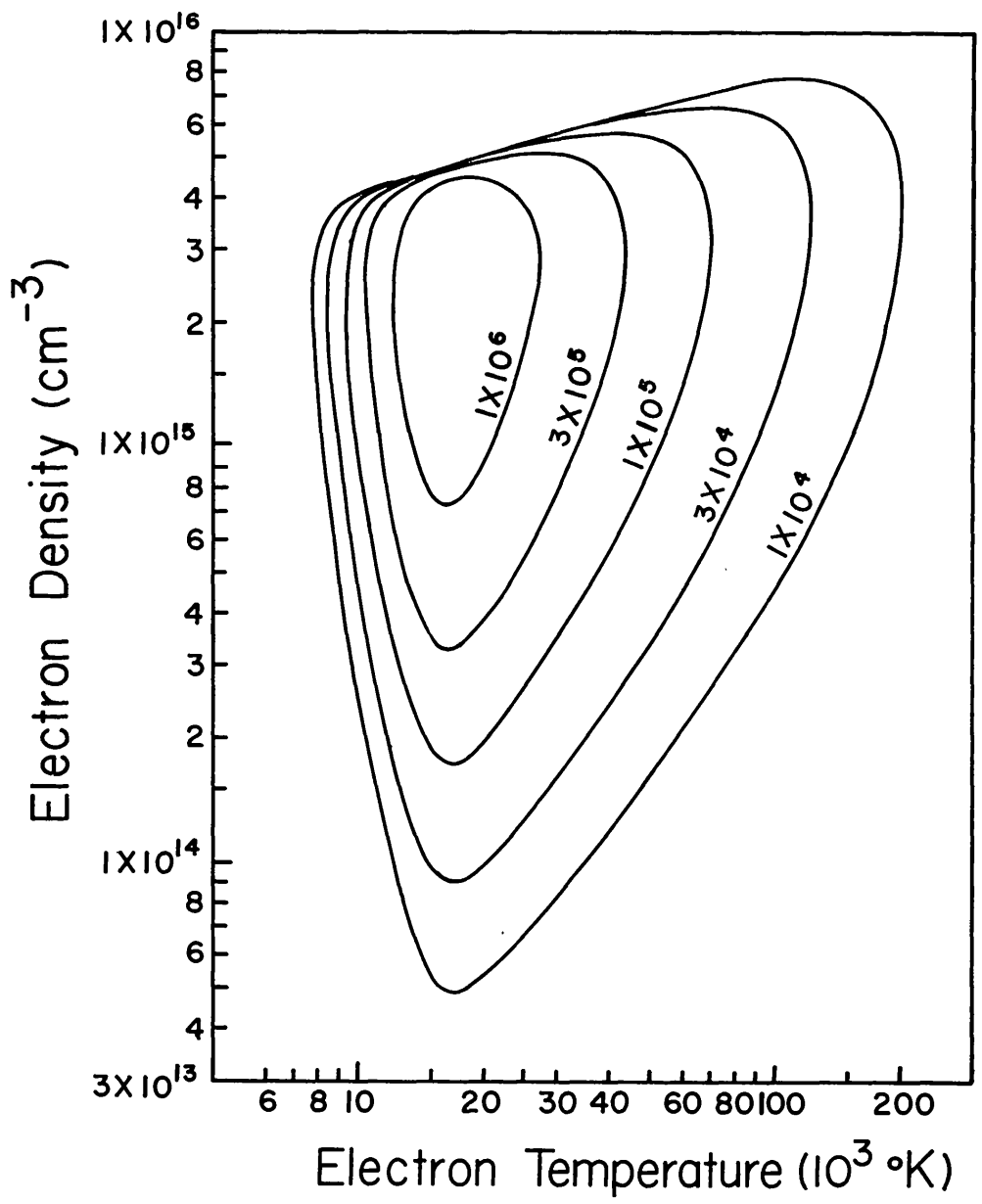

Fig. 1. Population inversion measure $P$ as a function of $n_{e}$ and $T_{e}$. The numbers by the side of contours represent $P$ values in $\mathrm{cm}^{-3}$.

be the sum of the contributions due to spontaneous emission and stimulated emission in varying proportions. It is obvious that if population inversion is taking place, the 'equivalent' temperature obtained from the Boltzmann formula will be negative. Also, intensity anomalies are expected.

\section{Application to 'Classical' Wolf-Rayet Stars}

It is well known that Wolf-Rayet stars show broad emission lines. It is generally believed (Pagel, 1960) that Beals's $(1930,1934,1940)$ hypothesis of the formation of emission lines in a rapidly expanding envelope is a reasonable explanation of the spectra of Wolf-Rayet stars. Beals $(1930,1934)$ assumed the operation of the Zanstra and Menzel mechanism for planetary nebulae, of photoionization with subsequent recombination, to be the source of emission lines. Beals's hypothesis is, however, strictly speaking only justified for great dilution of the incident radiation as compared with that of a black body, and it has been a theoretical puzzle (van Pelt, 1957) why it works 
for Wolf-Rayet stars, in the emission line region of which the dilution of radiation is not appreciable (Swings, 1942; Wilson, 1942). The considerations of the present paper provide the solution to this puzzle and also explain how $P>0$. In a supersonic expansion of an ionized gas, the dominant factor in describing the atomic processes is generally the recombination rate constant. The net result of the rapid cooling of electrons and subsequent recombinations is that it simulates the effects produced by dilute radiation. There is, however, one important difference. The $P$ values produced by the expansion mechanism for different transitions show a much greater range of magnitude than those produced by the dilute radiation mechanism. In other words, the expansion mechanism is much more selective in producing laser action than the dilute radiation mechanism.

A different approach has been followed by Aller (1943), who measured the emissionline intensities in a number of bright Wolf-Rayet stars. He attempted to estimate the temperature by using the relative intensities of two lines in conjunction with the Boltzmann formula. Let the measured intensities of two lines of a given ion be $I_{k i}$ and $I_{k^{\prime} i^{\prime}}$, their upper levels be $k$ and $k^{\prime}$ and their lower levels $i$ and $i^{\prime}$, respectively. If $N_{k}$ and $N_{k^{\prime}}$ are the populations of levels $k$ and $k^{\prime}$, respectively, then we have

$$
\frac{I_{k i}}{I_{k^{\prime} i^{\prime}}}=\frac{N_{k} A_{k i} h v_{k i}}{N_{k^{\prime}} A_{k^{\prime} i} h v_{k^{\prime} i^{\prime}}}
$$

Let $E_{k}$ and $E_{k^{\prime}}$ be the excitation potentials of $k$ and $k^{\prime}$; then making use of the Boltzmann formula, Equation (13) can be written as

$$
\frac{I_{k i}}{I_{k^{\prime} i^{\prime}}}=\frac{A_{k i} v_{k i} g_{k}}{A_{k^{\prime} i^{\prime}} v_{k^{\prime} i^{\prime}} g_{k^{\prime}}} \exp \left[-\left(E_{k}-E_{k^{\prime}}\right) / k T\right]
$$

For He II, Aller utilized the ratio of the intensities of the lines $\lambda \lambda 3203,4686$, and determined $T$. He obtained values ranging from $10000 \mathrm{~K}$ to $200000 \mathrm{~K}$ for different stars.

Weenen (1950) reanalysed Aller's data. Instead of determining $T$ from the ratio of emission intensities of certain pairs of lines, Weenen followed the method of Pannekoek and Doorn (1930) making use of all the available lines in one least-square solution. For the three WC stars for which Aller had carried out measurements, Weenen found from this procedure that $\mathrm{He}$ II lines led to negative temperatures. $\mathrm{He}$, however, did not appreciate the significance of this result and concluded that these temperatures have hardly any physical meaning. But it must be remembered that this was before the advent of lasers. Now, of course, we can readily infer from this that the negative temperatures imply population inversion in one or more pairs of levels. We demonstrate population inversions explicitly by the following simple procedure. Equation (3) shows that population inversion depends on the population per unit statistical weight, which we shall represent by $y_{k}$. Thus

$$
y_{k}=\frac{N_{k}}{g_{k}}=\frac{I_{k i}}{g_{k} A_{k i} h v_{k i}}
$$


TABLE I

Population per unit statistical weight for five levels of $\mathrm{He}$ II in three Wolf-Rayet stars. $n$ is the principal quantum number of the upper level, $I_{k i}$ is the measured intensity of the line (Aller, 1943), and $y_{k}$ is the population per unit statistical weight. The units of $I_{k i}$ and $y_{k}$ are arbitrary.

\begin{tabular}{|c|c|c|c|c|c|c|c|}
\hline \multirow{2}{*}{$\begin{array}{l}\text { Wavelength } \\
\text { (§) }\end{array}$} & \multirow[t]{2}{*}{$n$} & \multicolumn{2}{|c|}{ HD 192103} & \multicolumn{2}{|c|}{ HD 192641} & \multicolumn{2}{|c|}{ HD 193793} \\
\hline & & $\overline{I_{k i}}$ & $y_{k}$ & $\overline{I_{k i}}$ & $y_{k}$ & $\overline{I_{k i}}$ & $y_{k}$ \\
\hline 4686 & 4 & 73.6 & $3.77 \times 10^{3}$ & 35.5 & $1.82 \times 10^{3}$ & 43 & $2.20 \times 10^{3}$ \\
\hline 3203 & 5 & 41 & $3.76 \times 10^{3}$ & - & - & 11 & $1.01 \times 10^{3}$ \\
\hline 5412 & 7 & 10.1 & $5.77 \times 10^{3}$ & 5.4 & $3.09 \times 10^{3}$ & 18.5 & $1.06 \times 10^{4}$ \\
\hline 4859 & 8 & 11.2 & $9.40 \times 10^{3}$ & 2.6 & $2.18 \times 10^{3}$ & - & - \\
\hline 4542 & 9 & 10.0 & $1.18 \times 10^{4}$ & 4.1 & $4.85 \times 10^{3}$ & 11.7 & $1.38 \times 10^{4}$ \\
\hline
\end{tabular}

apart from a constant. The calculated values of $y_{k}$ for a number of levels of $\mathrm{He}$ II for the three WC stars in Aller's list are shown in Table I.

The population depends directly on the intensity. In drawing any conclusions from the results given in Table I, one must bear in mind the uncertainties in the recorded values of $I_{k i}$. We quote Aller (1943) on this point: 'It is extremely difficult to estimate the actual error in these intensities. The accidental error of a reasonably strong line measured on three or four plates is about $10 \%$, but this does not include systematic errors which may arise from estimating the continuous spectrum, for example. Weak lines, or lines in the far ultra-violet, may easily be in error by a full $100 \%$.'

We notice from Table I that for the star HD 192103 for which the lines are most intense, and the uncertainties are the least, the behaviour of $y_{k}$ is quite regular. Population inversion is seen to occur between any two of the levels having $n=5,7,8,9$ and also for transitions from $n=7,8,9$ to $n=4$. The other two stars also show population inversions for certain pairs of levels.

Beals (1934) and Aller (1943) have made intensity measurements of Wolf-Rayet bands, partly covering the same stars. As pointed out by Edlén (1956), the results are in some cases remarkably discordant. In the star HD 192103 both observers measured the bands $\lambda \lambda 5411,5470,5696,5812,5875$, all well defined and restricted to a fairly narrow wavelength region. Beals (1934) gives the following intensity figures: 22, 14 , $150,360,37$, while Aller's (1943) values are: $10,8,96,83,31$. If we assume a scale with 100 for $\lambda 5696$, the two sets of intensities can be expressed as follows, Beals: 14.7, 9.3, $100,240,24.7$, Aller: $10.4,8.3,100,86.5,32.3$. Notice the large discrepancy for $\lambda 5812$. Two possibilities exist: (a) measurement errors, and (b) the intensity of $\lambda 5812$ changed during the intervening period between Beals's and Aller's observations. The differences in the intensities of other lines in the two sets could probably be attributed to (a). However, for $\lambda 5812$ the difference is so large that (a) alone is possibly not enough to explain it and quite likely (b) is also a contributing factor. Should this be the case, then it is understandable on the basis of the model that we have discussed here. The line 
$\lambda 5812$ was very intense when Beals (1934) made his observations, probably laser action was contributing a major portion of the intensity. Small changes in $n_{e}$ and/or $T_{e}$ during the interval 1934-1943 could have significantly reduced the amount of laser action in this line, and Aller (1943) found it to be less intense.

It is known that in the WC stars the width of the emission lines increases from type WC9, where they are rather narrow, to WC5, where they are extremely broad. It would be reasonable to consider that the importance of laser processes increases from WC9 to WC5.

\section{Central Stars of Planetary Nebulae}

The central stars of planetary nebulae may be the ancestors of the white dwarfs (O'Dell, 1963; Harman and Seaton, 1964; Weidemann, 1968). Assuming this hypothesis to be correct, it would be reasonable to estimate that the electron densities in such objects will be intermediate between those existing in white dwarfs and those in the 'classical' Wolf-Rayet stars. The electron density in white dwarfs has been estimated to be about $2 \times 10^{16} \mathrm{~cm}^{-3}$ (Greenstein, 1958; Weidemann, 1963; Wiese and Kelleher, 1971). Thus the electron density in planetary nuclei is expected to be somewhat higher than that in the 'classical' Wolf-Rayet stars, and on the basis of Figure 1 we can conjecture that if $T_{e}$ values are appropriate, the population inversion for $\mathrm{He}$ II $\lambda 4686$ will be greater in planetary nuclei than that in the 'classical' WR stars.

Smith and Aller (1969) have given a classification scheme in which the emissionline central stars of planetary nebulae can be divided into five categories. One of their classes, comprising twelve initial members, is termed the $\mathrm{O}$ vi sequence. These stars have spectra in which the only really strong emission lines in the blue-green wavelength range are $\mathrm{He}$ II $\lambda 4686, \mathrm{C}$ IV $\lambda 4658$, and the O vI doublet $\lambda \lambda 3811,3834$. Subsequently, Sanduleak (1971) added five more stars to this sequence; these five stars do not appear to be planetary nuclei. In several stars amongst this total list of seventeen, the line broadening is quite large. These are the nuclei of NGC 6905, NGC 7026, NGC 5189, and the five stars in the list of Sanduleak (1971). These stars would be good candidates for investigating intensity anomalies in He II lines due to laser action.

\section{Terminology}

Finally, we would like to add some remarks as regards the terminology. The phenomenon that we have discussed is known, more specifically, as superradiance (Bloom, 1968) or superradiant emission (Lengyel, 1971; Siegman, 1971) in the literature of laser physics. Some authors (Allen and Peters, 1970; Allen, 1973) prefer the phrase amplified spontaneous emission. This helps to distinguish it from Dicke's superradiance and avoids the semantic confusion surrounding the word superradiance We have, however, used the more general term laser action to describe the phenomenon, because it is better known to astrophysicists and because Menzel has used it in the same 
sense. Also, the term maser action in the context of interstellar molecules is used in the same sense.

\section{Acknowledgement}

This work was supported in part by a research grant from the National Research Council of Canada.

\section{References}

Allen, L.: 1973, in L. Mandel and E. Wolf (eds.), Coherence and Quantum Optics, Plenum Press, New York.

Allen, L. and Peters, G. I.: 1970, Phys. Letters 31A, 95.

Aller, L. H.: 1943, Astrophys. J. 97, 135.

Ambartsumyan, V. A.: 1958, Theoretical Astrophysics (translated by J. B. Sykes), Pergamon Press, New York.

Bates, D. R. and Kingston, A. E.: 1963, Planet. Space Sci. 11, 1.

Bates, D. R., Kingston, A. E., and McWhirter, R. W. P.: 1962a, Proc. Roy. Soc. A267, 297.

Bates, D. R., Kingston, A. E., and McWhirter, R. W. P.: 1962b, Proc. Roy. Soc. A270, 155.

Beals, C. S.: 1930, Publ. Dominion Astrophys. Obs. Victoria 4, 271.

Beals, C. S.: 1934, Publ. Dominion Astrophys. Obs. Victoria 6, 95.

Beals, C. S.: 1940, J. Roy Astron. Soc. Canada 34, 169.

Bloom, A. L.: 1968, Gas Lasers, John Wiley \& Sons, Inc., New York.

Bohn, W. L.: 1971, Tenth Internat. Conf. on Phenomema in Ionized Gases, Donald Parsons \& Co., Oxford, p. 386.

Drawin, H. W.: 1967, Association Euratom-C.E.A. Report EUR-CEA-FC-383.

Drawin, H. W.: 1969, Z. Physik 225, 470.

Fujimoto, T., Sugiyama, I., and Fukuda, K.: 1972, Mem. Faculty of Eng. Kyoto Univ. 34, 249.

Green, L. C., Rush, P. P., and Chandler, C. D.: 1957, Astrophys. J. Suppl. 3, 37.

Greenstein, J. L.: 1958, Encyclopedia of Physics, Springer-Verlag, Berlin, 50, 161.

Gudzenko, L. I., Filippov, S. S., and Shelepin, L. A.: 1966, Zh. Eksp. Teor. Fiz. 51, 1115.

Gudzenko, L. I. and Shelepin, L. A.: 1963, Zh. Eksp. Teor. Fiz. 45, 1445.

Gudzenko, L. I., Shelepin, L. A., and Yakovlenko, S. I.: 1974, Usp. Fiz. Nauk 114, 457.

Hack, M. (ed.): 1968, Mass Loss from Stars, D. Reidel Publishing Co., Dordrecht.

Harman, R. J. and Seaton, M. J.: 1964, Astrophys. J. 140, 824.

Hoffmann, P. and Bohn, W. L.: 1972, Z. Naturforsch. 27a, 878.

House, L. L.: 1964, Astrophys. J. Suppl. 8, 307.

Irons, F. E. and Peacock, N. J.: 1974, J. Phys. B7, 1109.

Lengyel, B. A.: 1966, Introduction to Laser Physics, John Wiley, New York.

Lengyel, B. A.: 1971, Lasers, Wiley-Interscience.

McWhirter, R. W. P. and Hearn, A. G.: 1963, Proc. Phys. Soc. 82, 641.

Menzel, D. H.: 1937, Astrophys. J. 85, 40.

Menzel, D. H.: 1970, in H. G. Groth and P. Wellmann (eds.), Spectrum Formation in Stars with

Steady-State Extended Atmospheres N.B.S. Special Publication 332, p. 134.

O'Dell, C. R.: 1963, Astrophys. J. 138, 67.

Pagel, B. E. J.: 1960, Vistas in Astronomy 3, 203.

Pannekoek, A. and Doorn, N. W.: 1930, Verhandelingen Kon. Ned. Akad. v. Wetensch., Amsterdam 14, No. 2.

Sanduleak, N.: 1971, Astrophys. J. (Letters) 164, L71.

Seaton, M. J.: 1959, Monthly Notices Roy. Astron. Soc. 119, 81.

Siegman, A. E.: 1971, An Introduction to Lasers and Masers, McGraw-Hill Book Co., New York.

Smith, L. F. and Aller, L. H.: 1969, Astrophys. J. 157, 1245.

Sobolev, V. V.: 1960, Moving Envelopes of Stars (translated by S. Gaposchkin), Harvard University Press, Cambridge. 
Stevefelt, J. and Robben, F.: 1972, Phys. Rev. A5, 1502.

Swings, P.: 1942, Astrophys. J. 95, 112.

Underhill, A. B.: 1968, Ann. Rev. Astron. Astrophys. 6, 39.

van Pelt, A.: 1957, Bull. Astron. Inst. Netherlands 13, 285.

Varshni, Y. P. and Lam, C. S.: 1974, J. Roy. Astron. Soc. Canada 68, 264.

Weenen, J.: 1950, Bull. Astron. Inst. Netherlands, 11, 176.

Weidemann, V.: 1963, Z. Astrophys. 57, 87.

Weidemann, V.: 1968, Ann Rev. Astron. Astrophys. 6, 351.

Wiese, W. L. and Kelleher, D. E.: 1971, Astrophys. J. (Letters) 166, L59.

Willett, C. S.: 1974, Gas Lasers: Population Inversion Mechanisms, Pergamon Press, New York.

Wilson, O. C.: 1942, Astrophys. J. 95, 402.

Zemtsov, Yu. K.: 1969, Ninth Internat. Conf. on Phenomena in Ionized Gases, Academy of the Socialist Republic of Romania, Bucharest, p. 599. 